\title{
Autonomía escolar y cambio educativo, consideraciones desde la implementación de PEC-FIDE
}

\section{School Autonomy and Educational Change: Considerations in Light from the Implementation of PEC-FIDE}

\author{
Alejandro Reyes Juárez (*) alerejus@hotmail.com \\ (*) Subsecretaría de Educación Básica \\ (Recibido: 22 de febrero de 2014; Aceptado para su publicación: 18 de noviembre de 2015)
}

Cómo citar: Reyes, A. (2017). Autonomía escolar y cambio educativo, consideraciones desde la implementación del PEC-FIDE. Revista Electrónica de Investigación Educativa, 19(2), 12-21. https://doi.org/10.24320/redie.2017.19.2.643

\section{Resumen}

La SEP implementó un proyecto piloto denominado Programa de Fortalecimiento e Inversión Directa a las Escuelas (PEC-FIDE), cuyos propósitos consideraban: atender el problema de inequidad en el logro educativo y ofrecer una respuesta a las barreras en el acceso al financiamiento para comunidades vulnerables. El presente artículo es un ejercicio de análisis de la implementación de un programa educativo específico en el marco de un proceso de reforma que se desarrolla en México, es también una oportunidad para reflexionar en torno a temas como la autonomía escolar y el fortalecimiento de las competencias de gestión. La base es un estudio exploratorio que tuvo en algunos actores educativos (participantes en el PEC-FIDE) la principal fuente de información. Más que los recursos financieros distribuidos en las escuelas son las maneras en las que los recursos se aprovechan lo que abre posibilidades de transformación en algunas prácticas educativas y alternativas para atender las problemáticas particulares de las comunidades educativas.

Palabras clave: Política educativa, gestión escolar, autonomía escolar.

\section{Abstract}

The Secretariat of Public Education (SEP) implemented a pilot project known as the Program for Strengthening and Direct Investment in Schools (PEC-FIDE), the purpose of which was to address the problem of inequity in academic achievement and offer a solution to the obstacles in access to financing for vulnerable communities. This paper is an analysis of the implementation of a specific education program within the context of ongoing reform in Mexico, but also an opportunity to refelect upon topics such as school autonomy and the strengthening of management skills. The basis for this work is an exploratory study that drew its main source of information from educational actors who participated in the PEC-FIDE. It is not so much the financial resources themselves, but the ways in which these resources are used that open up possibilities of change in certain educational practices and alternatives to address specific problems in educational communities. 


\section{Introducción}

La evaluación docente como parte de los procesos de ingreso, promoción y permanencia en el servicio educativo ha constituido uno de los pilares de la reforma educativa implementada en los últimos años en México. Han quedado en un segundo plano aspectos como la evaluación interna contemplada en la misma Ley General del Servicio Profesional Docente (Secretaría de Educación Pública [SEP], 2013a) que entre otras cosas- implica el fortalecimiento en la práctica profesional de docentes y directivos, así como de los procesos de gestión escolar, lo que está en línea con lo establecido en el Programa Sectorial de Educación 2013-2018 (SEP, 2013b) sobre crear condiciones para que las escuelas ocupen el quehacer del Sistema Educativo y fortalezcan la capacidad de gestión de las escuelas como elementos para asegurar la calidad de los aprendizajes de la educación básica.

Es en este contexto que la discusión por la autonomía escolar parece retomar un nuevo auge. El impulso anterior se dio con el Acuerdo Nacional para la Modernización de la Educación Básica firmado hace más de dos décadas. En él ya se reconocía a la escuela como el centro del sistema educativo y a partir de éste se desarrolló un proceso de descentralización; el cual, aun con sus contradicciones, permitió impulsar la transformación de la gestión de los sistemas escolares (Arnaut, 2010).

La reforma actual y la de principios de la década de los noventa forman parte de las iniciativas de políticas educativas centradas en la escuela que han recorrido el continente americano y parte del mundo. En éstas se promueven procesos de descentralización y la gestión transita del conjunto del sistema al de cada uno de los componentes que lo integran, terminando en la escuela (Casassus, 2000). De esta manera la gestión educativa deja de ser una actividad exclusiva y propia de la cúspide del sistema educativo, produciendo redistribuciones de competencias entre los distintos actores, incluidos los que participan en los espacios escolares.

Este movimiento reformador de la gestión centrada en la escuela tiene en el Programa Escuelas de Calidad (PEC), implementado en México a partir de 2001, una de sus expresiones más sostenidas y que forma parte de los esfuerzos realizados en el país en ese sentido. El PEC promueve el empoderamiento de directores, maestros y padres de familia, al facultarlos para realizar ejercicios de planeación estratégica con el fin de mejorar el desempeño de la escuela, proporcionándoles recursos adicionales que posibilitan la puesta en marcha de planes de mejora (Murnane, Willett y Cárdenas, 2006). Se realiza, a través de las autoridades estatales, una aportación financiera directa a las escuelas y una contrapartida a las aportaciones que éstas logren reunir. Estos recursos han sido destinados, según las Reglas de Operación del PEC, en parte al fortalecimiento académico y formación continua de los colectivos escolares, así como a la adquisición de recursos materiales, mantenimiento e infraestructura.

Si bien es cierto que la mejora del aprendizaje no se estableció con claridad como propósito inicial del PEC (Murnane, Willett y Cárdenas, 2006), esto se ha convertido en uno de los elementos centrales de sus evaluaciones, las que han mostrado impactos positivos en las escuelas participantes en el programa: en los resultados del logro educativo y reducciones en las tasas de reprobación y deserción escolar. Sin embargo, son necesarios más datos para identificar con claridad los efectos atribuibles al programa y los factores que se interrelacionan para que esos efectos se den.

En el marco del Programa Escuelas de Calidad, durante el ciclo escolar 2008-2009, la SEP (en convenio con los gobiernos de Coahuila, Chihuahua, Quintana Roo, Hidalgo, Guanajuato y el Estado de México) implementó un proyecto piloto denominado Programa de Fortalecimiento e Inversión Directa a las Escuelas (PEC-FIDE), en cuyo impulso intervinieron organismos de la sociedad civil (Mexicanos Primero, Lazos por la educación y Fundación Idea); entre sus propósitos se consideraron: atender el problema de inequidad en el logro educativo; disminuir las tasas de deserción y ofrecer una respuesta a las barreras en el acceso al financiamiento para comunidades más vulnerables.

El proyecto tenía como componentes claves: la focalización para llegar a escuelas con altos niveles de insuficiencia y marginación; promover la planeación y el trabajo colectivo en la escuela, así como la participación social para detonar procesos de mejora educativa e inclusión escolar a través de una mejor alineación entre los recursos y las actividades escolares; la incorporación de zonas escolares completas y 
el fortalecimiento de la supervisión escolar. Retomó los instrumentos de operación del PEc, de manera particular la construcción de un Plan Anual de Trabajo (PAT) y un Plan Estratégico de Transformación Escolar (PETE). ${ }^{1}$ Contó con la colaboración del gobierno federal, estatal y sociedad civil, esta última como factor de contraloría social.

La conclusión del proyecto PEC-FIDE constituye una oportunidad para reflexionar sobre los procesos de gestión y autonomía escolar desde una experiencia en la que estos eran parte de sus propósitos. Además, en el proyecto se planteó también la importancia de estos procesos para mejorar las condiciones de aprendizaje de los estudiantes.

La autonomía de gestión escolar es entendida, dentro de los planteamientos de política educativa actual, como la capacidad de la escuela para tomar decisiones orientadas a mejorar la calidad del servicio educativo que ofrece. Esto es, que la escuela centra su actividad en el logro de aprendizajes de todos y cada uno de los estudiantes que atiende, garantizando con ello su derecho a la educación (SEP, 2014). De esta manera, la discusión sobre la autonomía escolar está relacionada con la capacidad de gobierno. Es decir, los márgenes de libertad que pueden gozar las instituciones educativas son concebidos como un producto de su capacidad de tomar decisiones de manera eficaz, considerando los contextos, problemas y necesidades particulares que los caracterizan.

El PEC-FIDE ha sido evaluado por los mismos organismos de la sociedad civil que lo impulsaron; Fundación Idea, de manera particular, evaluó su impacto y algunos aspectos de los cambios que generó (O’Donoghue, et al., 2009; Abreu, et al., 2010; O’Donoghue y Ongay, 2011).

El presente artículo se desprende de un ejercicio de indagación que sobre la implementación del PEC-FIDE se realizó a finales de 2013 por el equipo que daba seguimiento a la operación del Programa Escuelas de Calidad en la Subsecretaría de Educación Básica con el propósito de recuperar información, a través de los actores educativos, de los efectos de la implementación del proyecto de innovación PEC-FIDE sobre los procesos de gestión, autonomía escolar, equidad y calidad educativa. ${ }^{2}$ Para ello se seleccionaron, de los 212 planteles educativos contenidos en la base de datos, 2 escuelas por cada una de las entidades federativas que tuvieron una participación más prolongada en el proyecto: Estado de México, Hidalgo y Guanajuato. Para la selección se consideraron los dos niveles educativos (Primaria y Secundaria) y el tamaño de las escuelas, identificado a partir del número de grupos que atienden. Con estos aspectos como base, para contar con elementos contrastantes, en un segundo momento se realizó un muestreo aleatorio en cada una de las entidades federativas.

Se entrevistó a supervisores de las zonas escolares donde se ubicaban las escuelas, a los seis directores escolares de las escuelas participantes en el programa, a algunos profesores y padres de familia. Como ejemplo, se presentan algunas de preguntas planteadas a los directores:

- ¿Cómo fue el proceso de incorporación de la escuela al proyecto PEC-FIDE?

- ¿Cuáles son los mayores logros o avances que usted ha identificado en la escuela a partir de su participación en el proyecto PEC-FIDE?

- ¿Cuáles son los retos mayores que ha presentado para usted la participación de la escuela en el proyecto PEC-FIDE?

- ¿Qué instancias lo han apoyado durante el proceso de participación en el proyecto y cómo ha sido este apoyo?

- ¿Cómo han participado los padres de familia durante el desarrollo del proyecto?

- ¿Cómo elabora su PETE?

- ¿Cómo se decide qué adquirir con los recursos financieros que la escuela recibe con este proyecto?

\footnotetext{
${ }^{1}$ En la presente administración se plantea que la denominada Ruta de mejora constituya la herramienta de planeación colectiva escolar que pretende simplificar el proceso dentro de las instituciones educativas de educación básica.

${ }^{2}$ Participaron en este estudio exploratorio: Patricia Montero, Diana Murguía, Lilia Julieta Guzmán, Yajaira Santiago, Griselda Olmos y Adriana Navarrete.
} 
- ¿A quién y cómo informa sobre lo que se adquiere con los recursos financieros que aporta el proyecto PEC-FIDE?

- ¿Cuáles son las estrategias que han desarrollado para la atención de los estudiantes en riesgo?

- ¿Cuáles son las principales estrategias desarrolladas para la formación continua del colectivo escolar?

- ¿Si usted tuviera la decisión, que aspectos del PEC-FIDE llevaría a todas las escuelas y cuáles no?

\section{El cambio escolar}

Fullan y Stiegelbauer (2009) mencionan que el cambio educativo es un proceso multidimensional que, en última instancia, es una transformación de las prácticas. Las innovaciones articulan motivos políticos y educativos, así como realidades objetivas y subjetivas. De esta manera, las políticas y programas pueden ser más o menos específicos en términos de los cambios materiales, en las prácticas o en las convicciones que implican. Sin embargo, el punto decisivo está en las relaciones entre estos planteamientos y las realidades subjetivas construidas en los contextos particulares y como productos de las experiencias individuales. Se trata de crear las condiciones y los procesos que favorezcan la posibilidad de avanzar cada vez más hacia un mayor sentido de apropiación y compromiso (Fullan, 2007).

El PEC-FIDE fue concebido como un proyecto de innovación que pretendía promover algunos cambios en los procesos de gestión, para mejorar la "calidad educativa" de las escuelas que integran una misma zona escolar, en contextos de alta marginación y con altos niveles de rezago educativo. La distribución de recursos financieros directos a la escuela en razón de su matrícula y contemplando que un porcentaje del recurso sea dirigido a la atención de estudiantes con rezago educativo, además del impulso y fortalecimiento de la supervisión escolar, son las otras variables que lo distinguieron de la operación general del Programa Escuelas de Calidad.

El proyecto comenzó a operar en el ciclo escolar 2008-2009 en seis entidades: Chihuahua, Coahuila, Quintana Roo, Guanajuato, Hidalgo y Estado de México. Para 2009-2010, Chihuahua y Quintana Roo salieron del programa por las complicaciones para realizar la aportación financiera estatal, mientras Coahuila lo hizo en el ciclo escolar 2010-2011. Para 2011 el programa operaba en 215 escuelas de 16 zonas escolares en los tres estados que permanecieron, beneficiando a casi 25 mil estudiantes. El presupuesto anual fue de 8 millones de pesos por estado (4 millones de aportación federal y 4 millones aportados por cada entidad federativa). El monto otorgado por estudiante fue de 1023 pesos en el Estado de México, de 986 pesos en Guanajuato y 826 pesos en Hidalgo. Las escuelas recibieron en promedio 104600 pesos; el máximo fue de 561536 y el mínimo de 14790 pesos. Por su parte, los supervisores recibieron en promedio 43385 pesos.

Parece pertinente, antes de revisar algunos aspectos del proceso de implementación, agregar que los rubros o conceptos en que el recurso pudo invertirse fueron los siguientes: atención a alumnos en situación de riesgo educativo, al menos un 25\%; formación continua de directivos, docentes y padres de familia, hasta un 25\%; materiales y apoyos didácticos, equipamiento, construcción, mantenimiento o adecuación de espacios educativos, hasta un $30 \%$.

\subsection{Focalización, mejora en el logro educativo y atención al rezago}

No obstante que los criterios de selección estipularon la incorporación de escuelas ubicadas en contextos de alta marginación y con altos niveles de rezago educativo, en la práctica esto no sucedió en todos los casos. En promedio, el $80 \%$ de las escuelas PEC-FIDE se ubicó en zonas rurales y $20 \%$ en zonas urbanas. En Hidalgo la distribución fue de $60 \%$ y $40 \%$, respectivamente. Por lo que respecta a la ubicación en contextos de alta marginación, en Guanajuato el $74 \%$ de las escuelas se ubicó en municipios de alta o muy alta marginación; en el Estado de México el porcentaje fue parecido, con el 77\%; mientras que en Hidalgo la focalización fue más débil, ya que sólo el $49 \%$ de las escuelas participantes está ubicado en municipios de alta o muy alta marginación. 
Se puede agregar que el criterio de seleccionar zonas escolares completas (aunque esta regla tampoco se siguió al 100\% en algunos casos) contribuyó a que dentro de éstas coexistieran escuelas de diversas características socioeconómicas y de rezago educativo (O’Donoghue, et al., 2009; O’Donoghue y Ongay, 2011).

La evaluación realizada al PEC-FIDE mostró, entre otros resultados, que la tasa de aprobación en las escuelas participantes aumentó en promedio 1.41 puntos porcentuales. Por otro lado, en los resultados de la prueba ENLACE se observó que los estudiantes de primarias PEC-FIDE obtuvieron en promedio 20 puntos más en la prueba ENLACE de Español en tercer grado, por encima de las escuelas excluidas del programa. También se observó una reducción de 4 puntos porcentuales de alumnos que obtuvieron puntaje insuficiente en la misma prueba de Español para tercer grado. Los alumnos de tercero de secundaria incrementaron en 10 puntos su desempeño en la prueba ENLACE y se redujo en 5 puntos el porcentaje de alumnos en nivel insuficiente en esta prueba. Cuando se incluye en el análisis a las escuelas PEC-FIDE que previo al año 2008 estuvieron cubiertas por PEC, algunos efectos se desvanecen. En el caso de las primarias, el efecto en la prueba ENLACE de Español deja de ser significativo. En el caso de las secundarias, lo mismo es para la tasa de aprobación. Así, los efectos son más robustos en las escuelas que participan en PEC-FIDE por primera vez y, para el caso de las primarias, cuando hay un mejor proceso de focalización (Abreu et al., 2010).

A partir del análisis estadístico realizado por el equipo de la SEB, dentro del cual se realizó la comparación con las escuelas que participan en el PEC y las del Sistema Educativo Nacional, considerando los tres estados y modalidades educativas participantes en PEC-FIDE, se observó que si bien los indicadores de deserción y reprobación escolar tuvieron una disminución mayor en las escuelas que participaron en el PEC-FIDE, al convertir las poblaciones analizadas a puntuaciones z, para ser comparables, se observa que el PEC-FIDE presenta una tendencia similar al del Sistema Educativo Nacional en su conjunto.

Algo parecido ocurre al analizar los resultados de ENLACE. Al convertir las poblaciones analizadas a puntuaciones z, para ser comparables, se observa que el PEC-FIDE presenta, para Guanajuato e Hidalgo, la misma tendencia nacional, y en el caso del Estado de México para 2009 tiene un repunte en el número de estudiantes en bueno y excelente en Español. Sin embargo, en 2010 cae el porcentaje y posteriormente se comporta bajo la tendencia nacional. Para el caso de Matemáticas, al realizar el mismo proceso de convertir las poblaciones analizadas a puntuaciones z, para ser comparables, no se observan para el caso de Guanajuato e Hidalgo incrementos importantes en el porcentaje de alumnos en bueno y excelente de la prueba ENLACE diferentes a la tendencia nacional; sin embargo, en el caso del Estado de México el incremento en este porcentaje llega a superar hasta el del PEC en la entidad.

Al igual que con las evaluaciones realizadas al PEC, la información con que se cuenta no parece suficiente para identificar los efectos en la mejora del logro educativo y en la reducción de inequidades, atribuibles al proyecto PEC-FIDE, o cómo se interrelacionan los factores que hacen posibles buenos resultados.

De lo recuperado en las entrevistas realizadas con los actores educativos de las escuelas que participaron en el proyecto PEC-FIDE, se observó que los distintos actores identifican como uno de los objetivos PEC-FIDE elevar la calidad educativa, aunque ésta, coincidiendo con O’Donoghue et al. (2009), adquiere varios significados, que van desde la disponibilidad de mayores recursos educativos hasta mejores resultados educativos, pasando por la atención especial a los estudiantes con rezago, los procesos de formación continua y las transformaciones en las culturas escolares. En las escuelas que participaron en el proyecto PEC-FIDE las prácticas pedagógicas no parecen haberse transformado, son tradicionales pero en proceso de transición, los colectivos escolares integraron en su planeación escolar acciones específicas cuyo propósito era prevenir y atender a estudiantes en situación de riesgo de rezago educativo y deserción escolar. Lo anterior llevó, entre otras acciones, a la contratación de asesorías y talleres, de Matemáticas y Español para profesores y estudiantes. Entre las estrategias implementadas se establecieron algunas para atender e integrar a los padres de familia como un aspecto importante para conseguir un apoyo más amplio para los estudiantes.

Sin embargo, parece necesario desarrollar mecanismos para atender de manera diferenciada las problemáticas que colocan a los colectivos escolares en situación de riesgo. Y dentro de éstos, los 
estudiantes según sus necesidades particuares. Desde los resultados de la evaluación realizada por Fundación Idea, se concluye que aunque la atención del rezago educativo era uno de los componentes importantes del PEC-FIDE, incluso como un rubro específico al que se le destinaba un porcentaje del financiamiento, no hay claridad respecto a que esta estrategia -considerada importante dentro del proyecto- haya permeado hasta la escuela. Lo anterior se percibe en la poca claridad que había entre los actores educativos para definir el riesgo educativo y en las estrategias diversas para atender problemas de aprendizaje, conducta y salud que, por lo general, eran empleadas para todos los niños, independientemente del nivel de riesgo en el que se encontraban (O'Donoghue et al., 2009).

\subsection{Gestión, autonomía y supervisión}

Más que los recursos financieros distribuidos en las escuelas, es la forma en que estos son aprovechados por los colectivos escolares lo que parece abrir las posibilidades de transformaciones en las prácticas educativas y alternativas para atender las problemáticas particulares. En este contexto, el papel de los directivos escolares es relevante. Navarro (2015) plantea al respecto que, no obstante que la dirección tiende a perpetuar las prácticas instituidas dentro de las organizaciones, abre la posibilidad de generar espacios en donde tienen lugar el cambio y la mejora.

La importancia del director coincide con lo presentado por O’Donoghue y Ongay (2011) en la evaluación del PEC-FIDE, cuando mencionan que la gestión en las escuelas es un trabajo en el que intervienen diversos actores escolares, pero el director se constituyó en la piedra angular del proyecto y es identificado como el líder de éste. Sin embargo, desde lo identificado por la investigación que da sustento al presente artículo, son las habilidades de gestión de cada director y las perspectivas centradas en lo pedagógico que posee -un producto de una formación que va más allá de la implementación del proyecto- lo que permite la potencialización de los recursos asignados.

Entre otras cosas, lo anterior posibilita: la adquisición de recursos para apoyar el aprendizaje y la enseñanza; el fomento de su uso entre docentes y estudiantes; y la apertura de espacios de diálogo y reflexión entre profesores y padres de familia para buscar respuestas a los problemas educativos y atender a los estudiantes.

El proyecto en sí tiene muchas bondades, nos da mucho margen para ser autogestivos no sólo en cuanto a los resultados, sino en la forma de trabajar y de acomodar cada centavo. Creo que da ese margen de ponernos de acuerdo entre nosotros, y con los papás, ante la situación de la escuela... Nos da mucha autonomía en poder decidir qué es lo que más le conviene a la escuela, siempre, siempre poniendo en primer término el servicio a los niños (Director de Primaria).

Yo veía la toma de decisiones más restringida. Pero, viéndolo con los compañeros, da más resultados PEC-FIDE porque no deja orillarte... la toma de decisiones está más centrada a atacar un problema que tenga que ver con el aprendizaje de los alumnos. Y PEC es más amplio (Director de Telesecundaria).

En el caso de PEC-FIDE han sido considerados recursos específicos para la atención de estudiantes con rezago educativo, a pesar de las complicaciones para la identificación de éste (señaladas en el apartado anterior) contribuye para que estas decisiones tengan presente de manera constante el aprendizaje y la atención de los estudiantes.

En algunos casos, a decir de profesores y padres de familia, los cambios se observaron a la llegada del director, aunque la escuela ya estuviera participando en el proyecto PEC-FIDE con anticipación. De la misma manera, en algunas escuelas -según la información recuperada en las entrevistas- la salida del director escolar que comenzó el proyecto provocó un estancamiento o que los logros alcanzados se vieran amenazados. En este sentido, se puede decir que la inversión ejercida por las escuelas en formación continua fue significativa. Sin embargo, la movilidad de docentes y directivos redujo el impacto de las acciones en beneficio de la comunidad escolar. 
En general, se puede concluir que la entrega de recursos financieros de manera directa a las escuelas da al colectivo escolar un mayor margen para decidir sobre las necesidades identificadas y las estrategias para atenderlas. Además, se fortaleció la participación de los diversos actores, incluidos los padres de familia, sobre todo en los procesos de transparencia y rendición de cuentas.

Los supervisores también recibieron apoyo financiero, pero las conclusiones de las evaluaciones realizadas por Fundación indican que los cambios en estos actores escolares no fueron visibles, ni fueron alcanzadas las metas planteadas a este nivel (O'Donoghue et al, 2009). No obstante, se empezaron a desarrollar algunas redes de apoyo -no como parte de una estructura sino de manera más informalentre las escuelas y los actores escolares (O’Donoghue y Ongay, 2011).

Se registró también que, al igual que en las escuelas, en las zonas escolares la disponibilidad de recursos amplía el margen de gestión y la toma de decisiones. En algunos casos se constató que la incorporación de las escuelas de la misma zona detona un mayor compromiso del supervisor, mayor cercanía y acompañamiento para impulsar el liderazgo directivo, mientras en otros casos permitió a los supervisores impulsar el trabajo en redes para solucionar problemas y atender necesidades comunes. Sin embargo, al igual que sucedió con los directores escolares, la información recuperada permite concluir que más que el recurso designado a las supervisiones escolares, son las habilidades de cada supervisor para la gestión lo que potencializa las acciones de mejoramiento en la zona escolar.

\subsection{Distribución, ejercicio y comprobación de recursos}

La posibilidad de que las escuelas, principalmente las ubicadas en contextos vulnerables por la pobreza y marginación que enfrentan, cuenten con recursos adicionales para atender necesidades particulares detectadas por las propias comunidades educativas, lo cual a su vez fortalece en alguna medida los procesos de gestión y autonomía, parece una medida pertinente con los principios de calidad y equidad establecidos en la legislación educativa. En general se observó una evaluación positiva (por parte de los distintos actores educativos) de los recursos a los que se tuvieron acceso. El monto de los recursos recibidos permitió a las escuelas desarrollar acciones para fortalecer el aprendizaje de los estudiantes, además de adquirir materiales y recursos educativos diversos, así como realizar acciones de mantenimiento y dignificación de los espacios educativos.

Sin embargo, la aportación directa a las escuelas, dentro de la experiencia de quienes participaron en el PEC-FIDE se enfrentó a una serie de problemáticas que generaron algunos cuestionamientos. O’Donoghue y Ongay (2011) registraron que la preocupación mayor tenía relación con la repartición por porcentajes en los distintos componentes establecidos y con la regla de dar recurso por alumno. Lo cual resultaba en algunos casos inequitativo y restaba efectividad al proyecto. En el mismo sentido, los datos recuperados por el equipo de la SEB dieron cuenta de una serie de necesidades que no se pueden atender con el recurso del proyecto; por ejemplo, no quedó claro el tipo de inversión que puede hacerse en infraestructura. Se solicitó flexibilizar los porcentajes y los rubros de gasto, ya que dependiendo del proceso en el que la escuela esté, serán diferentes las prioridades a atender.

Se planteó la necesidad de establecer criterios de equidad (o compensatorios) en la asignación del recurso a las escuelas con menor matrícula. Y, desde la perspectiva de los actores, la entrega de recursos fue tardía y desfasada del ciclo escolar. Fueron distintos los mecanismos que se emplearon en las entidades federativas para hecer llegar los recursos a las escuelas; desde el depósito en cuentas bancarias hasta la organización de exposiciones de proveedores donde las escuelas seleccionaban lo que requerían, y las autoridades educativas estatales realizaban el pago y atendían el proceso de facturación. En general, se observó que la entrega de recurso directo a las escuelas implica una excesiva carga administrativa para los colectivos escolares. Y los catálogos y ferias de proveedores no ofertaron todos los bienes y servicios que cubrieran en su totalidad las necesidades de las escuelas.

En cuanto a la transparencia y rendición de cuentas, se identificó un fortalecimiento de estos mecanismos. Aunque no todos los actores escolares conocen el origen de los recursos, en algunos casos mencionaron que fueron proporcionados por organizaciones de la sociedad civil participantes en el diseño y seguimiento del proyecto, cuando no fue así. 


\section{Consideraciones finales}

La equidad sigue siendo el gran reto del Sistema Educativo Nacional. Parece haber coincidencia en cuanto a que en la explicación de las desigualdades de los resultados educativos, los factores socioculturales, la pobreza y la marginación tienen un papel relevante (López, 2008; Blanco, 2011; UNICEF, 2012). Pero también que entre las barreras que producen o agravan estas inequidades, y la exclusión, hay algunas que se configuran desde la escuela y dentro del sistema educativo (UNICEF, 2012).

De esta manera, la escuela -apoyada por el sistema educativo en su conjunto-constituye un frente desde donde se puede contribuir de manera relevante a generar cambios que enfrenten la reproducción de las inequidades. Esto sin olvidar que la identificación, dentro de las políticas centradas en la escuela, de las instituciones escolares como espacios relevantes para construir respuestas a los problemas educativos del país, ha llevado a cargar de demandas a las escuelas y a los actores escolares. Se les responsabiliza, en cierta medida, de los pobres resultados educativos y los funcionamientos deficientes, sin un pertinente fortalecimiento de sus competencias para enfrentarlos, sin que sus perspectivas, intereses y necesidades hayan sido adecuadamente recuperados en las políticas; muchas veces excluidos de la definición de los problemas y de la construcción de las políticas.

Se sabe que las políticas cobran sentido, se cuestionan y resignifican en las prácticas cotidianas que se desarrollan en las escuelas, como producto de las interrelaciones entre sujetos e instituciones. Las escuelas traducen las propuestas, las interpretan, las quiebran encontrando intersticios en los que despliegan su autonomía aprovechando sus potencialidades de formas diversas; pero también las momifican, las fetichizan, las banalizan. Por esto, las políticas no son, exclusivamente, las que definen o deciden los cambios reales, sino el modo en que colectivamente se apropian y reelaboran (Frigerio, 2000). Por ello es necesario que la escuela construya una capacidad interna de desarrollo, ya que sin ésta (aun cuando tenga los oportunos apoyos externos) la mejora educativa será siempre algo marginal, no sostenible en el tiempo (Bolívar, 2009).

Se requiere de procesos de evaluación de programas y políticas más constantes e integrales que sean referente para adecuaciones y rediseños en busca de la mejora educativa, y acortar distancias entre el diseño de las políticas públicas y lo implementado. A pesar de esta acotación, lo analizado en el presente artículo parece corresponder con los planteamientos de la necesidad de fortalecer las competencias internas de la escuela.

Si bien los recursos canalizados directamente a la escuela abren la posibilidad de atender necesidades particulares identificadas por las comunidades educativas, el factor clave para que esto se potencialice son las perspectivas centradas en lo educativo y las competencias de gestión y liderazgo de algunos de los actores escolares, principalmente del director. Lo que se ve reproducido a nivel de la supervisión escolar.

La autonomía de gestión, contemplada en la más reciente Reforma Educativa, como medio para mejorar la calidad educativa, representa un reto mayor. Constituye uno de los referentes de diversos programas en marcha como el Programa de la Reforma Educativa (anteriormente Programa Escuelas de Excelencia para Abatir el Rezago Educativo), el Programa Escuelas de Tiempo Completo y el propio Programa de Escuelas de Calidad, además de las acciones realizadas para fortalecer los Consejos Técnicos Escolares y la Supervisión Escolar, lo que abre una posibilidad en la construcción de innovaciones educativas desde los propios centros escolares (Gather, 2004).

Sin embargo, promoverla, garantizarla, desarrollarla y fortalecerla será un proceso complicado, que requiere algo más que inversiones directas a las escuelas. En última instancia ésta ha sido una forma de solventar parte de los requerimientos que las comunidades escolares necesitan para desarrollar los procesos educativos de forma eficaz, equitativa y pertinente; en esencia es una política de compensación.

La disponibilidad de recursos para atender necesidades específicas, y las decisiones que las comunidades educativas tomen sobre su uso, debe ser sólo uno de los ámbitos en los que la autonomía escolar debe ejercerse, sin perder de vista que la formación de los estudiantes en general, y los aprendizajes del plan de estudios en particular, son la prioridad. La autonomía, como incremento de la capacidad de toma de 
decisiones a nivel de centro, debe pasar del decreto a la construcción de condiciones para que sea posible: dispositivos; competencias, apoyos, asistencia y asesoramiento; articulación de esfuerzos y corresponsabilidad; procesos de seguimiento y evaluación que, como menciona Bolívar (2005) permitan que las escuelas, en conjunción con su entorno local, puedan construir su propio espacio de desarrollo, en función de unos objetivos asumidos en un proyecto colegiadamente.

En esta línea, la evaluación interna y la formación continua de los directivos escolares (que considera la Ley del Servicio Profesional Docente) parecen claves en el fortalecimiento de la autonomía de gestión de las escuelas de educación básica, aun con los cuestionamientos que a ésta se han realizado (Smyth, 2001). La autonomía como un mecanismo para garantizar el derecho a la educación de los niños y jóvenes mexicanos, no como un abandono a la suerte de las comunidades educativas, lo que reproduciría y ampliaría las brechas educativas y sociales.

\section{Referencias}

Arnaut, A. (2010). Gestión del sistema educativo federalizado 1992-2010, En A. Arnaut y S. Giorguli (Coords.), Los grandes problemas de México. El Colegio de México.

Abreu, R. et al. (2010). Evaluación de impacto del proyecto piloto PEC-FIDE. México: Fundación Idea.

Blanco, E. (2011). Los límites de la escuela: educación, desigualdad y aprendizajes en México. El Colegio de México.

Bolívar, A. (2009). De 'la escuela no importa' a la escuela como unidad base de mejora. punto.edu, 5(15), 28-33. Recuperado de http://www.cipes.org/articulos/1115\%20-\%20De\%20la\%20escuela.pdf

Bolívar, A. (2005). ¿Dónde situar los esfuerzos de mejora?: política educativa, escuela y aula. Educ. Soc., Campinas, 26(92), 859-888.

Casassus, J. (2000). Problemas de la gestión educativa en América Latina (la tensión entre los paradigmas de tipo A y el tipo B). Recuperado de http://www.lie.upn.mx/docs/Especializacion/Gestion/Lec2\%20.pdf

Frigerio, G. (2000). ¿Las Reformas Educativas Reforman las Escuelas o las escuelas Reforman las Reformas? Santiago de Chile: UNESCO-ORELAC.

Fullan, M. (2007). Las fuerzas del cambio con creces. Madrid: Akal.

Fullan, M. y Stiegelbauer, S. (2009). El cambio educativo. Guía de planeación para maestros. México: Trillas.

Gather, M. (2004). Innovar en el seno de la institución escolar. Barcelona: Graó.

López, N. (Coord.) (2008). Políticas de equidad educativa en México. Análisis y propuestas. Buenos Aires: IIPEUNESCO.

Murnane, R., Willett, J. y Cárdenas, S. (2006). ¿Ha contribuido el Programa Escuelas de Calidad (PEC) a mejorar la educación Pública en México? Aprender más y mejor. Políticas, programas y oportunidades de aprendizaje en educación básica en México. México: Harvard-SEP-FCE-ILCE.

Navarro, C. (2015). La antesala del ascenso a la dirección. Trayectorias de subdirectores de secundarias mexicanas. México: comie.

O’Donoghue, J. et al. (2009). Evaluación cualitativa del proyecto piloto PEC-FIDE. México: Fundación Idea.

O’Donoghue, J. y Ongay, E. (2011). Evaluación del proyecto PEC-FIDE. México: Fundación Idea. 
Secretaría de Educación Pública [SEP] (2010). Programa Escuelas de Calidad. Módulo I. Modelo de gestión educativa estratégica. Recuperado de

http://Formacion.sigeyucatan.gob.mx/formacion/materiales/5/d3/p3/3.\%20EL\%20MODELO\%20DE\%20G ESTION\%20EDUCATIVA\%20ESTRATEGICA.pdF

SEP (2014). Acuerdo número 717 por el que se emiten los lineamientos para formular los Programas de Gestión Escolar. Diario Oficial de la Federación. México, Segob.

SEP (2013a). "Decreto por el que se expide la Ley General del Servicio Profesional Docente". Diario Oficial de la Federación. Recuperado de

http://www.dof.gob.mx/nota detalle.php?codigo=5313843\&fecha=11/09/2013

SEP (2013b). Programa Sectorial de Educación 2013-2018. México: Autor.

Smyth, J. (Ed.) (2001). La autonomía escolar. Una perspectiva crítica. Madrid: Akal.

UNICEF (2012). Completar la escuela un derecho para crecer, un deber para compartir. Recuperado de http://www.siteal.iipe-oei.org/sites/default/files/informe-unesco-unicef-ago-2 